\title{
Inconclusive evidence on systemic treatments for recurrent aphthous stomatitis
}

\author{
Abstracted from \\ Brocklehurst P, Tickle M, Glenny AM, et al. \\ Systemic interventions for recurrent aphthous stomatitis (mouth ulcers). \\ Cochrane Database Syst Rev. 2012; 9: CD005411.pub2. \\ Address for correspondence: Luisa Fernandez Mauleffinch, Review Group Co-ordinator, \\ Cochrane Oral Health Group, MANDEC, School of Dentistry, University of Manchester, \\ Higher Cambridge Street, Manchester, M15 6FH, UK. E-mail: luisa.fernandez@manchester.ac.uk
}

\section{Question: How effective are systemic treatments for recurrent aphthous stomatitis (RAS)?}

Data sources The trial registers of the Cochrane Oral Health and Pain, Palliative and Supportive Care Groups were searched together with The Cochrane Central database, Medline, Embase, CINHAL and AMED databases. Reference lists of relevant articles were also searched and authors of trials contacted.

Study selection Randomised controlled trials (RCTs), including those with cross-over design where the primary outcome was a reduction of pain associated with RAS, a reduction in episode duration or episode frequency were included.

Data extraction and synthesis Data were extracted independently by two authors and risk of bias assessment was conducted on six domains. Cochrane statistical guidelines were to be followed.

Results Twenty-five trials were included; 22 were placebo controlled and eight made head-to-head comparisons (five trials had more than two treatment arms). Twenty-one different interventions were assessed. The interventions were grouped into two categories: immunomodulatory/anti-inflammatory and uncertain. Only one study was assessed as being at low risk of bias. There was insufficient evidence to support or refute the use of any intervention.

Conclusions No single treatment was found to be effective and therefore the results remain inconclusive in regard to the best systemic intervention for RAS. This is likely to reflect the poor methodological rigour of trials, and lack of studies for certain drugs, rather than the true effect of the intervention. It is also recognised that in clinical practice, individual drugs appear to work for individual patients and so the interventions are likely to be complex in nature. In addition, it is acknowledged that systemic interventions are often reserved for those patients who have been unresponsive to topical treatments, and therefore may represent a select group of patients.

This paper is based on a Cochrane Review published in the Cochrane Library 2012, issue 9 (see www.thecochranelibrary.com for information). Cochrane Reviews are regularly updated as new evidence emerges and in response to feedback, and the Cochrane Library should be consulted for the most recent version of the review.

\section{Commentary}

This review addresses a less common approach for the management of recurrent aphthous stomatitis (RAS) with no underlying systemic cause, since most clinicians rely on topical treatments.

In most cases RAS is self-limiting and multifactorial in nature. The location of the painful ulcers and level of recurrence varies from individual to individual and can greatly affect quality of life. Common treatments mainly target pain reduction with different degrees of success depending on the patient. Topical measures do not affect the frequency of recurrences. The authors performed an extensive search for randomised controlled trials electronically and by hand. The search also included unpublished papers with no language restrictions. The primary outcomes evaluated were pain, duration, frequency, safety and adverse events. Secondary outcomes were quality of life improvement and increase in function. Risk of bias was assessed on the 25 studies that met the inclusion criteria. The risk of bias was low in one study, unclear for eight and high risk for the remainder.

The researches separated the interventions according to the nature of the agent used between immunomodulator/anti-inflammatory agents and uncertain. Eight different agents belong to the first category in which levamisole, a drug labelled for use for cancer, was the most studied. Eight trials compared active ingredients. Nine different interventions were evaluated in the uncertain category.

Overall the individual studies were too different in aspects like timing and outcome measure to allow for any comparison. Adding the limited quality, no recommendation for any agent was possible.

Regardless of the high quality of the review, the modest number of subjects and the restricted length of the included studies limits the ability to identify potential long-term benefits or complications.

Possible interactions with other medications will need to be considered. Potential gaps in the present understanding of RAS may explain the limited efficacy of some modalities.

Despite the results, it is important to note that most of the agents described are drugs or interventions that are not part of the clinician 's everyday armamentarium and as a result may not be adopted even in the presence of more compelling evidence.

\section{Practice point \\ - No recommendations for the use for any particular systemic agent for the treatment of recurrent aphthous stomatitis (RAS).}

Silvia Spivakovsky

NYU College of Dentistry, New York, USA

Evidence-Based Dentistry (2012) 13, 120. doi:10.1038/sj.ebd.6400901 